\title{
Basophil activation test in the diagnostics of hymenoptera venom allergy
}

\author{
Nemsovska $\mathrm{J}^{1}$, Waczulikova $\mathrm{I}^{2}$, Svecova $\mathrm{D}^{1}$ \\ Department of Dermatovenerology, Faculty of Medicine, Comenius University in Bratislava \\ and University Hospital Old Town, Bratislava, Slovakia. nemsovska.jana@gmail.com
}

\begin{abstract}
OBJECTIVES: The aim of the study was to analyse the diagnostic performance of the basophil activation test (BAT), to compare the diagnostic reliability of BATs performed with different types of allergens, which are available in Slovakia and to verify the correlation between the symptom severity and the sensitivity and specificity of the BAT in 114 patients with suspected hymenoptera venom allergy (HVA).

BACKGROUND: Diagnosis of the HVA and the identification of the appropriate venom for immunotherapy are in Slovakia based on detailed patient'medical history, skin tests and detection of slgE. In unclear cases, where the clinical decision regarding the relevant insect species for immunotherapy is difficult, the cellular tests are recommended in several countries, such as Sweden, Spain, Germany, Denmark and Italy. In Slovakia, BAT is not adapted as s part of routine diagnostic work-up.

METHODS: The identification of the culprit hymenoptera species among 114 patients was based on detailed history, skin tests and detection of slgE. Obtained results were compared with the results acquired by the BAT. RESULTS: The sensitivity of the BAT was $80.8 \%$ and the specificity was $87.8 \%$. The sensitivity of the BAT was higher when using Soluprick SQ Allergens, but the specificity was higher with BÜHLMANN CAST Allergens. In the study no correlation between the symptom severity and the sensitivity and specificity of the BAT was observed.

CONCLUSIONS: The results show that the BAT can be recommended in the identification of the appropriate venom for immunotherapy, the only specific treatment that is currently available for patients with HVA. Allergen source is one of critical factors in diagnostic reliability of the BAT (Tab. 4, Ref. 29). Text in PDF www.elis.sk KEY WORDS: hymenoptera venom allergy, allergy diagnosis, basophil activation test, sensitivity, specificity.
\end{abstract}

\section{Introduction}

Hymenoptera venom allergy (HVA) is the most common cause of anaphylactic reactions in adults and the second-most common cause in children, after food-related anaphylaxis $(1,2)$. In European epidemiological studies the sensitization rate to honeybee or wasp venom in the general population varies between $27.1-44.1 \%$ (3). Strong local reactions occur in 2.4 to $26.4 \%$ of general population. The prevalence of systemic reactions ranges between 0.3 and $7.5 \%$ in adult population (4). The estimated number of annual mortalities due to insect sting-induced anaphylaxis ranges from 0.03 to 0.45 per one million inhabitants (5) Due to the fear of future reactions, HVA imposes a significant impact in healthrelated Quality of Life $(6,7)$.

\footnotetext{
${ }^{1}$ Department of Dermatovenerology, Faculty of Medicine, Comenius University in Bratislava and University Hospital Old Town, Bratislava, Slovakia, and ${ }^{2}$ Department of Nuclear Physics and Biophysics, Faculty of Mathematics, Physics and Informatics, Comenius University, Bratislava, Slovakia

Address of correspondence: J. Nemsovska, MD, MPH, MA, Department of Dermatovenerology, Faculty of Medicine, Comenius University in Bratislava and University Hospital Bratislava, Mickiewiczova 13, SK-813 69 Bratislava, Slovakia.

Phone: +421.2 .57290651$

E-mail: jana.nemsovska@sm.unb.sk
}

The management of HVA includes short-term interventions to treat acute reactions and long-term strategies to minimize the risk of consecutive reactions. Acute reactions are managed by symptomatic treatment. Long-term care includes patient education on how to minimize exposure to further stings, prescription of an emergency kit for self-treatment and venom immunotherapy (VIT) (8). VIT is the only specific treatment that is currently available for patients with a history of systemic reaction to a hymenoptera insect sting (9). VIT with the responsible hymenoptera venoms is highly effective in protecting patients with hymenoptera venom allergy including anaphylaxis. It has proven to be effective in $95 \%$ of patients allergic to wasp venom and between 75 and $85 \%$ of those allergic to honeybee venom (10). Identification of the appropriate venom for VIT is often hampered by a high frequency of double sensitizations to honeybee and wasp venom. Depending on the method used, up to $64 \%$ of insect venom-allergic patients show double-positivity. Double sensitization could be caused by true double sensitizations, cross-reactive specific $\operatorname{IgE}(\mathrm{sIgE})$ antibodies that recognize similar protein epitopes of hyaluronidase, vitellogenin or dipeptidylpeptidase of honeybee and wasp venom, or the presence of SIgE to cross-reactive carbohydrate determinations (CCD) in both venom allergens (11). It is pondered that IgE directed to CCD is the major cause of multiple sensitization detection (12). Real double sensitization to both venoms indicates 
potential systemic allergic reactions to the next sting by either insect species, if not treated by immunotherapy with both venoms. In the case of cross-reactions as a cause for double positivity, the treatment with the venom of the primarily responsible insect alone would be sufficient and more cost effective (13).

In Western and Central Europe sting reactions are primarily due to sting by certain social wasps (Vespula vulgaris and Vespula germanica in particular) and to honeybees (Apis mellifera). Paper wasp species are very common in Southern Europe, the US and in tropical and subtropical areas (especially South America) $(1,14)$. The identification of the appropriate venom for VIT is based on detailed history, venom skin tests and detection of hymenoptera sIgE. The determination of sIgE by component-resolved diagnostic test (CRD) allows a more precise characterization of the sensitization profile of an individual patient. Cellular tests, like the basophil activation test (BAT), are recommended in unclear cases where the decision regarding the relevant insect species for immunotherapy is difficult.

The BAT reports both high sensitivity (85-100\%) and specificity $(83-100 \%)$ to diagnose hymenoptera venom allergy $(15,16)$. Its high specificity is based on monitoring cellular response following antigen stimulation in real time (10). Basophils with their high-affinity IgE receptors are easily accessible and, therefore, they can be used as indicator cells for IgE-mediated reactions. Upon allergen challenge and cross-linking of membrane-bound IgE antibodies, basophils up-regulate certain activation markers on their surface, such as CD63 and CD203c, as well as intracellular molecules, such as p38 mitogen activated protein kinase (17). The BAT uses flow cytometry to measure the expression of these activation markers.

The aim of the following prospective study was to analyse the diagnostic reliability of the BAT among selected patients with suspected hymenoptera venom allergy. Furthermore, the study compares the diagnostic performance of BAT performed with different allergens, which are available in Slovakia: commercial venom extracts for prick testing Soluprick SQ, ALK-Abelló, Denmark and BÜHLMANN CAST Allergens, Germany, optimized for in vitro use in the cellular allergy assays. In addition, the study compares the sensitivity and specificity of BAT performed with honeybee venom and BAT performed with wasp venom, and assess the correlation between the severity of symptoms and the sensitivity and specificity of the BAT.

\section{Patients and methods}

A total of 114 consecutive patients (47 men, 67 women) between the ages of 6 and 72 years (average age $47.5 \pm 14.66$ years) with suspicion of HVA were evaluated in the Outpatient Department of Dermato-allergology, Department of Dermatovenerology, University Hospital, Bratislava, Slovakia between 2014 and 2019. Laboratory diagnostic trsts were performed in the Department of Clinical Immunology and Allergology, Oncology Institute St. Elizabeth, Bratislava, Slovakia.

Of the 114 evaluated patients 81 had a documented history of systemic allergic reaction (SR) after hymenoptera sting as fol- lows: 38 experienced a grade I reaction, 23 experienced a grade II reaction, 14 experienced a grade III reaction, and 6 of them experienced a grade IV reaction, according to H. L. Mueller (18). 24 patients experienced strong local reactions. Nine patients had no reaction after the sting. All of them were participants in a beekeeping course, which required the results of allergic testing against hymenoptera allergens.

The time interval from the last sting to examination was between 1 and 52 months (average time interval 10.2 \pm 3 months) for 85 patients. The exact time interval was not given in 20 patients (time interval 10 or more years). Nine patients, participants in a beekeeping course, had no history of stings in the past.

All individuals enrolled in the study were informed and signed the informed consent. The approval number from the Ethical committee of the Old Town Hospital and the Faculty of Medicine of Comenius University, Bratislava, Slovak Republic is 25/2021.

The identification of the culprit hymenoptera species was based on detailed history, venom skin tests and detection of hymenoptera venom-specific IgE. A combination of these tests improved the diagnostic precision in HVA (10). The results of these tests were compared with the results of the BAT. Skin testing and blood sampling for in vitro testing were realized in one session.

In particular, patients with systemic mastocytosis or patients with high serum tryptase level $(>11.4 \mathrm{ng} / \mathrm{mL})$ are at an increased risk of frequent and severe episodes of anaphylaxis, therefore in all involved patients the basal serum tryptase level was determined (19).

\section{Patient's history}

All patients were questioned for severity of symptoms and, in particular, for the type of insect responsible for the sting. The verification of a previous reaction after sting by clinical history should build the basis for subsequent diagnostic work-up since asymptomatic sensitization to Hymenoptera venom is observed frequently (5).

\section{Skin tests}

In all patients, skin sensitivity to both venoms (honeybee and wasp) was determined by a skin prick test (SPT). In several countries skin testing is considered the gold standard, because they are easy to conduct, fast and relatively inexpensive and can provide helpful diagnostic information $(20,21)$. SPTs were performed with commercial venom extracts for prick testing Soluprick SQ, ALK-Abelló, Denmark. The venoms, honeybee venom (Apis mellifera) and wasp venom (Vespula species) were tested. Histamine dihydrochloride $10 \mathrm{mg} / \mathrm{mL}$ and physiological saline were used as positive and negative controls, respectively. Testing was done with incremental concentrations of $10 \mu \mathrm{g} / \mathrm{mL}, 100 \mu \mathrm{g} / \mathrm{mL}$ and 300 $\mu \mathrm{g} / \mathrm{mL}$. Positive results were defined as a wheal $\geq 3 \mathrm{~mm}$ diameter with a flare 15-20 minutes after the SPT. No systemic reactions have occurred following SPTs.

\section{Determination of specific immunoglobin E antibodies (sIgE)}

Detecting SIgE in conjunction with the patients's clinical history is one of the main diagnostic methods in HVA (20). In all patients, 


\section{8-784}

sIgE to whole insect venoms of honeybee, Vespula spp. (Yellow jacket), Vespa crabro (Hornet), Polistes spp. (European wasp), Dolichovespula arenaria and Dolichovespula maculata (Bald-faced hornet) were determined by standardized solid phase enzyme immunoassay ELISA(Enzyme-linked immunosorbent assay), Hytec 288, Hycor, USA. The recombinant venom allergens used were rApi $\mathrm{m} 1$ (phospholipase A2 Apis mellifera), rVes v 1 (phospholipase A1 - Vespula vulgaris), rVes v 5 (antigen 5 - Vespula vulgaris) and the MUXF3 carbohydrate epitope from bromelain. Bromelain extracted from pineapples is a glycoprotein commonly used for reference to sIgE-CCD detection and analysis. The diagnosis was established by the use of FEIA(Fluorescent enzyme immunoassay), ImunoCAP Phadia250 (Thermo Fisher), Sweden. A value of $0.35 \geq \mathrm{kU} / \mathrm{L}$ was considered positive.

\section{Basophil activation test}

The BAT was performed with the kit BasoFlowEx Kit, Exbio, Czech Republic. In the first 84 cases, the allergens used were commercial venom extracts (Soluprick SQ, ALK-Abelló, Denmark) with bee venom (Apis mellifera) and wasp venom (Vespula species), all in 3 different concentrations $(10 \mu \mathrm{g} / \mathrm{mL}, 100 \mu \mathrm{g} / \mathrm{mL}$ and $300 \mu \mathrm{g} / \mathrm{mL})$. In the next 30 cases, the allergens used were BÜHLMANN CAST Allergens, Germany: BAG2-I1 Honey Bee Venom and BAG2I3 Yellow Jacket Venom, both in a final concentration of $11.5 \mathrm{ng} / \mathrm{mL}$. The analysis was performed on BD FACSCanto II flow cytometer, Becton Dickinson Bioscience, San Jose, California, USA.

The analyses were performed within 8 hours of blood sampling to heparinized tubes. One hundred $\mu \mathrm{L}$ of the whole blood was incubated with $100 \mu \mathrm{L}$ of stimulation buffer (BasoFlowEx Kit) containing IL-3 to enhance the allergen-specific up-regulation of CD63, and $10 \mu \mathrm{L}$ of allergen for $15 \mathrm{mi}-$ nutes at $37^{\circ} \mathrm{C}$. The positive control was prepared with stimulation buffer and $10 \mu \mathrm{L}$ of the stimulation control (BasoFlowEx Kit) containing chemotactic peptide fMLP (N-formyl-methionyl-leucyl-phenylalanine). Negative control contained only the stimulation buffer. Subsequently 20 $\mu \mathrm{L}$ of Staining Reagent (BasoFlowEx Kit) containing monoclonal antibodies was added to each sample. The antibodies included were anti-CD63 labelled with fluorochrome FITC (Fluorescein isothiocyanate) for measuring basophil activation and anti-CD203c Allergens.
Tab. 1. Diagnostic performance analysis of BAT.

\begin{tabular}{|c|c|c|c|}
\hline & BAT bee & BAT wasp & BAT total \\
\hline Prevalence $^{\mathrm{a}}(\%)^{\mathrm{b}}$ & $13.6 \quad(7.6-20.8)$ & $55.3 \quad(45.7-64.6)$ & $64.0 \quad(54.5-72.8)$ \\
\hline PPV ( \%) & $(27.8-74.3)$ & $83.9 \quad(72.3-91.9)$ & $(82.7-97.4)$ \\
\hline NPV ( \%) & $(89.4-98.2)$ & $78.8 \quad(65.3-88.9)$ & $(57.5-83.8)$ \\
\hline Sensitivity ( $\%$ ) & $(44.9-92.2)$ & $82.5 \quad(70.9-90.9)$ & $(69.9-89.1)$ \\
\hline Specificity ( \%) & $89.9 \quad(82.2-95.1)$ & $80.4 \quad(66.9-90.2)$ & $87.8 \quad(73.8-95.9)$ \\
\hline $\mathrm{LR}+$ & $7.26(3.68-13.86)$ & $4.21(2.50-7.56)$ & $6.63(3.13-15.3)$ \\
\hline LR- & $0.29(0.12-0.58)$ & $0.22(0.12-0.36)$ & $0.22(0.13-0.34)$ \\
\hline DOR & 24.47 & 19.38 & 30.34 \\
\hline
\end{tabular}

${ }^{\text {a }}$ Prevalence in the target population of patients with clinical suspicion of HVA.

${ }^{\mathrm{b}}$ All proportions are expressed as percentages together with $95 \%$ confidence intervals

BAT bee-BAT performed with honeybee venom (irrespective of the type of allergen: Soluprick or BÜHLMANN), BAT wasp - BAT performed with wasp venom (irrespective of the type of allergen: Soluprick or BÜHLMANN), BAT total - results of BAT irrespective of the type of allergen (bee venom or wasp venom, Soluprick or BÜHLMANN), PPV - Predictive value of +ve test (post-test likelihood of disease), NPV - Predictive values of -ve test (post-test likelihood of no disease), LR+ - Likelihood Ratio positive test, LR- - Likelihood Ratio negative test, DOR - Diagnostic Odds Ratio.

Tab. 2. Diagnostic performance analysis of BAT performed with Soluprick SQ allergens.

\begin{tabular}{lclclc}
\hline Soluprick SQ & \multicolumn{2}{c}{ BAT bee } & \multicolumn{2}{c}{ BAT wasp } & \multicolumn{2}{c}{ BAT total } \\
\hline Prevalence $^{\mathrm{a}}(\%)^{\mathrm{b}}$ & 14.3 & $(7.6-23.6)$ & 52.4 & $(41.2-63.4)$ & $64.7(49.5-71.2)$ \\
PPV (\%) & 52.6 & $(28.8-75.5)$ & 80.0 & $(66.3-89.9)$ & $90.2(78.6-96.7)$ \\
NPV (\%) & 96.9 & $(89.3-99.6)$ & 88.2 & $(72.5-96.7)$ & $84.8(68.1-94.9)$ \\
Sensitivity (\%) & 83.3 & $(51.6-97.9)$ & 90.9 & $(78.3-97.5)$ & $90.2(78.6-96.7)$ \\
Specificity (\%) & $87.5(77.6-94.1)$ & $75.0(58.8-87.3)$ & $84.9(68.1-94.9)$ \\
LR+ & $6.67(3.39-12.83)$ & $3.64(2.23-6.45)$ & $5.95(2.89-15.3)$ \\
LR- & $0.19(0.05-0.51)$ & $0.12(0.05-0.29)$ & $0.11(0.05-0.25)$ \\
DOR & 35.0 & 30.0 & \multicolumn{2}{c}{51.5} \\
\hline
\end{tabular}

${ }^{\text {a }}$ Prevalence in the target population of patients with clinical suspicion of HVA.

${ }^{\mathrm{b}}$ All proportions are expressed as percentages together with $95 \%$ confidence interval

BAT bee - BAT performed with honeybee venom, BAT wasp - BAT performed with wasp venom, BAT total BAT performed with Soluprick SQ allergens (irrespective of the type of allergen: bee venom or wasp venom), PPV - Predictive value of +ve test (post-test likelihood of disease), NPV - Predictive values of -ve test (posttest likelihood of no disease), LR+ - Likelihood Ratio positive test, LR- - Likelihood Ratio negative test, DOR - Diagnostic Odds Ratio.

Tab. 3. Diagnostic performance analysis of BAT performed with BÜHLMANN CAST

\begin{tabular}{|c|c|c|c|}
\hline BÜHLMANN CAST & BAT bee & BAT wasp & BAT total \\
\hline Prevalence $^{\mathrm{a}}(\%)^{\mathrm{b}}$ & $(2.1-26.5)$ & $(43.8-79.9)$ & $(53.9-87.5)$ \\
\hline PPV ( \%) & $(1.3-98.7)$ & $(72.4-100.0)$ & $(74.2-100.0)$ \\
\hline NPV ( \%) & $(76.5-99.1)$ & $(35.7-82.7)$ & $(22.9-72.2)$ \\
\hline Sensitivity ( $\%$ ) & $(0.8-90.6)$ & $(38.4-83.7)$ & $(36.4-79.3)$ \\
\hline Specificity ( \%) & $(81.0-99.9)$ & $99.1 \quad(70.3-100.0)$ & $(61.6-100.0)$ \\
\hline $\mathrm{LR}+$ & $9.0 \quad(0.99-69.27)$ & $70.1 \quad(2.25-2839.2)$ & $47.8 \quad(1.67-1938.4)$ \\
\hline LR- & $0.69(0.21-1.0)$ & $0.37(0.19-0.61)$ & $0.41(0.23-0.70)$ \\
\hline DOR & 13.0 & 188.6 & 115.5 \\
\hline
\end{tabular}

a Prevalence in the target population of patients with clinical suspicion of HVA.

${ }^{\mathrm{b}}$ All proportions are expressed as percentages together with $95 \%$ confidence interval

BAT bee - BAT performed with honeybee venom, BAT wasp - BAT performed with wasp venom, BAT total BAT performed with BÜHLMANN CAST Allergens (irrespective of the type of allergen: bee venom or wasp venom), PPV - Predictive value of + ve test (post-test likelihood of disease), NPV - Predictive values of -ve test (post-test likelihood of no disease), LR+ - Likelihood Ratio positive test, LR- - Likelihood Ratio negative test, DOR - Diagnostic Odds Ratio. The results may be affected by small sample size.

labelled with fluorochrome PE (Phycoerythrin) for gating the basophil population as well as measuring basophil activation. After staining, the samples were incubated for 20 minutes at $4{ }^{\circ} \mathrm{C}$, lysed for 10 minutes (Lysing Solution, BasoFlowEx Kit), centrifuged at $300 \mathrm{xg}$ for 5 minutes and washed. After decanting the supernatant, the samples were re-suspended in $300 \mu \mathrm{L}$ of PBS (Phosphatebuffered saline) and analysed within 2 hours using flow cytometry. 
The result of the BAT was considered positive, when more than $10 \%$ of basophils were activated (basophil reactivity $\geq 10 \%$ ).

\section{Statistical analysis}

All participants' data were statistically described, analyzed for between-group differences, and summarized as means with respective standard deviations (SD) for continuous variables, or in contingency tables as counts or percentages. Agreement analysis was used to evaluate the degree of agreement and disagreement between the tested BAT method and the reference ELISA method over categories in $2 \times 2$ tables. Chi-square tests were used to test the agreement, disagreement and significant differences. Strength of agreement was expressed using the unweighted Cohen's kappa which measures the agreement between two sets of ratings discounting any element of agreement expected to have arisen by chance. A kappa above 0.8 indicates a very good agreement beyond chance. The McNemar's statistic was used to test for asymmetry in the distribution of samples (subjects) where the ratings disagreed. The diagnostic performance, accuracy, sensitivity, specificity, positive predictive value (PPV), and negative predictive value (NPV) of the screening test were calculated. All estimates were presented along with the respective $95 \%$ confidence intervals (95\% CI).

Statistical analyses were performed using StatsDirect 3.2.7 software (Stats Direct Ltd., Cheshire, UK). All reported P values were two-sided, and significance was set at $\mathrm{p}<0.05$.

\section{Results}

In total, 114 subjects with suspicion of Hymenoptera venom allergy were enrolled; $41 \%$ (47 patients) were males and 59\% (67 patients) were females. The average age was $47.5 \pm 14.66$ years, median age was 50 years (range 6-72 years), lower quartile 35.5 and upper quartile 61.5 years.

Based on the results of patient's history, SPT, measurement of sIgE to whole insect venoms and molecular allergy diagnostics among the study population, 10 patients $(8.8 \%)$ were found to be allergic to honeybee venom, 58 patients $(50.8 \%)$ were allergic to wasp venom, 5 patients $(4.4 \%)$ were allergic to both venoms and 41 patients $(36 \%)$ were not allergic to honeybee or wasp. Five patients $(4.4 \%)$ had elevated serum tryptase level $(>11.4 \mathrm{ng} / \mathrm{mL})$.

The BAT was performed with two different allergens. In the first 84 patients $(73.7 \%)$ commercial venom extracts for prick testing Soluprick SQ, ALK-Abelló, Denmark were used; the sample of each patient was examined with both honeybee and wasp venom, both in 3 different concentrations. In the next 30 patients (26\%), BÜHLMANN CAST Allergens, Germany were used, the sample of each patient was examined with both honeybee and wasp venom, both in one concentration recommended by the manufacturer.

1. Agreement and disagreement analyses of the BAT performed with honeybee venom and the BAT performed with wasp venom (irrespective of the type of allergen: Soluprick or BÜHLMANN): In honeybee testing the general agreement in $87.7 \%$ was observed and chance-adjusted agreement ex-
Tab. 4. Diagnostic performance analysis of BAT depending on symptom severity.

\begin{tabular}{|c|c|c|c|c|}
\hline & & $\begin{array}{c}\text { No reaction } \\
+\mathrm{LLR}\end{array}$ & $\begin{array}{c}\text { SR Grade I } \\
+ \text { Grade II } \\
\end{array}$ & $\begin{array}{l}\text { SR Grade II } \\
+ \text { Grade IV }\end{array}$ \\
\hline \multirow{8}{*}{ BAT bee } & Prevalence $^{\mathrm{a}}(\%)^{\mathrm{b}}$ & 0.62 & 16.1 & 25.0 \\
\hline & PPV ( \%) & 2.44 & 69.2 & 50.0 \\
\hline & NPV ( \%) & 99.6 & 97.9 & 81.2 \\
\hline & Sensitivity ( \%) & 50.0 & 90.0 & 40.0 \\
\hline & Specificity ( \%) & 87.5 & 92.3 & 86.7 \\
\hline & $\mathrm{LR}+$ & 4.0 & 11.7 & 3.0 \\
\hline & LR- & 0.57 & 0.11 & 0.69 \\
\hline & DOR & 7.0 & 108.0 & 4.33 \\
\hline \multirow{8}{*}{ BAT wasp } & Prevalence $^{\mathrm{a}}(\%)^{\mathrm{b}}$ & 19.0 & 64.5 & 85.0 \\
\hline & PPV ( \%) & 54.5 & 89.2 & 92.9 \\
\hline & NPV ( \%) & 99.5 & 72.0 & 33.33 \\
\hline & Sensitivity ( $\%$ ) & 98.4 & 82.5 & 76.5 \\
\hline & Specificity ( \%) & 80.8 & 81.8 & 66.7 \\
\hline & $\mathrm{LR}+$ & 5.11 & 4.54 & 2.29 \\
\hline & LR- & 0.02 & 0.21 & 0.35 \\
\hline & DOR & 252.0 & 21.21 & 6.5 \\
\hline
\end{tabular}

Prevalence in the target population of patients with clinical suspicion of HVA.

${ }^{\mathrm{b}}$ All proportions are expressed as percentages together with $95 \%$ confidence interval BAT bee - BAT performed with honeybee venom, BAT wasp - BAT performed with wasp venom, No reaction + LLR - the group of patients with no reaction and patients with LLR (33 patients - $28.9 \%$ ), SR Grade I + Grade II - group of patients experienced a grade I or grade II reaction (61 patients $-53.5 \%$ ), SR Grade III + Grade IV - group of patients experienced a grade III or grade IV reaction (20 patients -17.5 ), PPV - Predictive value of +ve test (post-test likelihood of disease), NPV - Predictive values of -ve test (post-test likelihood of no disease), LR+ - Likelihood Ratio positive test, LR- - Likelihood Ratio negative test, DOR - Diagnostic Odds Ratio. The results in some categories may be affected by small sample size

pressed as Cohen's kappa was 0.54 (95\% CI 0.33 to 0.75 ; p $<0.0001)$. Disagreement and its asymmetry was not significant $(\mathrm{p}=0.109)$.In wasp testing the general agreement was in $81.58 \%$, Cohen's kappa of 0.63 (95\% CI 0.48 to 0.77 ; p $<0.0001)$. No significant disagreement was observed $(\mathrm{p}=$ 0.827). Diagnostic performance analysis of the BAT is summarized in Table 1.

2. Agreement and disagreement analyses of the BAT performed with Soluprick SQ allergens that were used in 84 patients (73.7 \%): In honeybee testing, the general agreement was observed in $86.9 \%$ and Cohen's kappa was 0.56 (95\% CI 0.35 to $0.79 ; \mathrm{p}<0.0001)$. Here, a significant asymmetric disagreement $(\mathrm{p}=0.0348)$, with a higher proportion of false positives (9) than false negatives was identified (2). These discordant proportions, however, were low in absolute values (in favor of high proportions of concordant ratings), so generalizability of the findings of disagreement beyond the sample is limited. In wasp testing the general agreement was observed in $83.33 \%$ and Cohen's kappa was 0.66 (95\% CI 0.50 to $0.82 ; p<0.0001)$. Disagreement was not significant $(\mathrm{p}=0.109)$. Diagnostic performance analysis of the BAT performed with Soluprick SQ allergens are summarized in Table 2.

3. Agreement and disagreement analyses of the BAT performed with BÜHLMANN CAST Allergens that were used in 30 patients $(26 \%)$. The results may be affected by the small sample size. In honeybee testing we observed the general agreement in $90 \%$ and Cohen's kappa was 0.35 (95\% CI -0.22 to 0.92 ; $\mathrm{p}=0.0255)$. Disagreement was not significant $\mathrm{p}=0.564$. In 


\section{8-784}

wasp testing the general agreement was observed in $76.97 \%$ with Cohen's kappa of 0.56 (95\% CI 0.29 to $0.81 ; p=0.0003)$. Disagreement with significant asymmetry $(\mathrm{p}=0.0082)$ was detected with higher proportion of false negatives (7) than false positives (0). These proportions were low in absolute values, which does not allow drawing any conclusions that extend beyond the sample studied. Diagnostic performance analysis of the BAT performed with BÜHLMANN CAST Allergens are summarized in Table 3.

4. Diagnostic performance analysis of the BAT based on severity of symptoms. Eighty-one patients (71\%) had a documented history of SR after hymenoptera: 38 of them experienced a grade I reaction (33.3\% of all patients), 23 of them experienced a grade II reaction ( $20 \%$ of all patients), 14 of them experienced a grade III reaction (12\% of all patients), and 6 of them experienced a grade IV reaction ( $6 \%$ of all patients). Twenty-four patients $(21 \%)$ had a large local reaction and 9 patients $(7.9 \%)$ had no reaction. Diagnostic performance analyses of the BAT depending on severity of symptoms are summarized in Table 4.

\section{Discussion}

The basophil activation is emerging as a reliable and robust in vitro biomarker of allergy reactions occurring in vivo (10). In the past, testing the basophil response to allergen was focused on the measurement of mediators released by cells in the supernatant in vitro. This testing has largely been replaced by the BAT after the discovery of the up-regulation of CD63 during basophil activation in $1991(16,20)$.

Activation marker CD63 is a membrane protein localized in the same secretory lysosomal granule that contains histamine; therefore, histamine release and up-regulation of CD63 correlate well during activation of basophils. Where histamine is thought to be released by both piecemeal degranulation and anaphylactic degranulation, CD63 is a precise marker of anaphylactic degranulation through regulated exocytosis after allergen-mediated activation of basophils as well as mast cells. CD203c is frequently measured in addition to CD63 and appears to co-express with CD63 even though the pathways for up-regulation differ. CD203c highly reflects piecemeal degranulation $(16,22)$. The BAT uses the flow cytometry to measure the expression of these activation markers.

There are two common measures of basophil activity: basophil reactivity and basophil sensitivity. Basophil reactivity refers to the number of basophils that respond to a given stimulus and basophil sensitivity to the allergen concentration at which half of all reactive basophil respond (16).

The BAT is essentially an assay with superior specificity compared to any other allergy testing. If appropriately used, it can provide a valuable clinical asset in different areas of allergy diagnostics $(23,24,25)$. Measurement and comparison of basophil sensitivity during the allergen immunotherapy can improve treatment response monitoring $(10,15)$. A successful VIT, which necessarily induces long term tolerance, decreases BAT sensitivity, without changing the reactivity (5).
In the present study, the culprit allergen was established based on the patient's history, skin prick tests and determination of SIgE to the whole insect venoms and their individual allergens (rApi m 1 , rVes v 1 , rVes v 5) as well as SIgE to MUXF3 by the molecular allergy diagnostics. The sensitivity of skin prick test alone is around $64 \%$. The sensitivity of sIgE detection to yellow jacket venom for yellow jacket venom-allergic patients ranges between $83 \%$ and $97 \%$. The sensitivity of sIgE detection to honeybee venom for honeybee venom- allergic patients ranges between $90 \%$ and $100 \%$. Diagnostic sensitivity of a combination of two commercially available yellow jacket venom allergens Ves $\mathrm{v} 1$ and Ves v 5 ranges between $92 \%$ and $100 \%$. The first commercially available honeybee venom allergen Api $\mathrm{m} 1$ yielded a diagnostic sensitivity of $58 \%$ to $97 \%$ (5). The combination of these tests improves the diagnostic precision in HVA and the identification of the appropriate venom for VIT.

The challenge testing was not performed. However, challenge testing is the gold standard for diagnosis of food or drug-induced anaphylaxis. A sting challenge with a live insect should not be used in patients with HVA who are not desensitized, because of the high risk of anaphylaxis. The allergen dose cannot be incrementally increased. Moreover, such sting challenge tests are not helpful in patients with a history of prior anaphylaxis, because the absence of early systemic symptoms does not exclude the possibility of a later systemic reaction $(4,8)$. Current recommendations advocate a sting challenge under an ongoing VIT only. A controlled sting challenge is considered to be the gold standard to evaluate the clinical response of the patient, allowing a reliable conclusion about the success of VIT (26). Despite maximum safety precautions and standby of emergency management, there is a potential of severe anaphylaxis, therefore sting challenge with a live insect is not perform routinely (25).

Although the BAT is not part of the routine diagnostics of venom allergy in all patients, it is well established and can be used in cases of unclear or negative skin and SIgE test results or when clinical history and diagnosis are contradictory. Studies demonstrated that BAT is able to detect sensitization in $80 \%$ of venomallergic patients with negative $\operatorname{sgE}$ and in $60 \%$ of patients that additionally exhibit negative intradermal skin tests (5). A study of the paediatric population revealed that the BAT allows for the identification of the culprit insect with higher specificity than the IgE reactivity test (27). The BAT has been suggested to be helpful in distinguishing a real double sensitization and cross-reactivity due to sequence homology among venom proteins. In addition, the BAT can be helpful in patients with systemic masocytosis or patients with high serum tryptase levels. In these patients total $\operatorname{IgE}$ and sIgE levels can be lover than in the general population. In general, these sIgE levels are below the traditional cutoff value, in which case there is difficulty in diagnosing HVA (28).

The diagnostic sensitivity of the BAT with insect venoms referred to the history was found to be $85-100 \%$ and the diagnostic specificity was $83-100 \%(15,16)$. The sensitivity for the BAT measuring CD63 expression is reported as $89 \%$ and $97 \%$ for CD203c expression, but the use of the CD63 is more widespread $(15,16)$. In the present study, both activation markers (CD203c, 
CD63) were measured to improve the sensitivity of BAT; CD203c was also used for gating the basophil population $(10,16)$.

Allergen source is one of critical factors in both clinical and research applications of the BAT. Allergen stimulants range from crude extracts to recombinant or purified single allergen sources. Standardized allergen reagents are recommended when comparing performance data from different laboratories and when performing tests over time. The availability of recombinant allergens for the BAT may be limited but they have the greatest stability and consistency compared to crude allergen or extracts and can help improve diagnostic accuracy $(15,16,20)$.

The present study found the sensitivity of the BAT to be $80.8 \%$ and the specificity to be $87.8 \%$. The prevalence was $64.0 \%$, the PPV was $92.2 \%$ and the NPV was $72.0 \%$. The obtained sensitivity $(80.8 \%)$ was, in general, lower than previously reported values $(85-100 \%)$; the obtained specificity $(87.8 \%)$ confirmed literary data $(15,16,17)$. The sensitivity, specificity, prevalence, PPV, and NPV obtained in the BAT with commercial venom extracts for SPT Soluprick SQ were 90.2 \%, 84.9\%, $64.7 \%, 90.2 \%, 84.8 \%$, respectively. The sensitivity, specificity, prevalence, PPV, and NPV obtained in the BAT with BÜHLMANN CAST Allergens were $59.1 \%, 98.8 \%, 73.1 \%, 99.2 \%, 47.1 \%$, respectively.

The resulting value of BAT sensitivity was affected by the result of sensitivity of the BAT performed with BÜHLMANN CAST Allergens, which was much lower (51.9 \%) than the sensitivity of the BAT performed with commercial venom extras for SPT Soluprick SQ (90.2\%), although BÜHLMANN CAST Allergens were optimized for in vitro use in the cellular allergy assays. The low sensitivity of the BAT with BÜHLMANN CAST Allergens may be attributable to the use of only one concentration of allergen recommended by manufacturer $(11.5 \mathrm{ng} / \mathrm{mL})$ whereas 3 different concentrations of Soluprick SQ Allergens (10, 100 and $300 \mu \mathrm{g}$ ) were used. Moreover, the results of the BAT with BÜHLMANN CAST Allergens could also be a result of the small sample size.

On the contrary, the specificity was higher for the BAT with BÜHLMANN CAST Allergens (98.8\%). The lower sensitivity of the BAT with Soluprick SQ Allergens (84.9\%) may have been caused by the use of allergens for SPT instead of allergens for intradermal tests (IDT), which are commonly used for the BAT (10). The allergen extracts for SPT are aqueous solutions of proteins combined with $50 \%$ glycerol, which acts as a preservative. The solutions are, therefore, quite viscous. Glycerol is not included in IDT extracts as it may trigger false positive results during the intradermal testing. Included components and physical properties of SPT extracts may affect results of BAT specificity. Therefore, the allergen extracts for IDT are more respectable for BAT than allergen extracts for SPT $(10,29)$. In our study, we did not have the option to use allergen extracts for IDT because they are not available in Slovakia.

We found that the sensitivity of the BAT with wasp venom was higher (82.5\%) than the BAT with honeybee venom (73.3 \%); conversely, the BAT with honeybee venom demonstrated higher specificity $(89.9 \%)$ than the BAT performed with wasp venom $(80.4 \%)$.
Our study did not confirm any association between the symptom severity and the sensitivity and specificity of BAT. This study is limited by the small number of cases in some categories according to severity of symptoms.

Our results confirmed that the BAT is a respectable method used in controversial cases of HVA reflecting high specificity and lower sensitivity. Therefore, the BAT is a confirmation test used for identifying the appropriate venom for VIT. For screening, the tests with high sensitivity are acceptable, such as skin tests and detection of SIgE. The sensitivity can be further increased by combining of these tests (10). In our study, we demonstrated the high specificity of BÜHLMANN CAST Allergens that are optimized for in vitro use in the cellular allergy assays. The sensitivity of the test with BÜHLMANN CAST Allergens may be increased by using different concentrations of allergens.

\section{Conclusions}

The BAT using flow cytometry can be helpful in the identification of the appropriate venom for VIT when used in combination with other well-established tests. Also, BAT could be recommended as a confirmatory test in the identification of the appropriate venom for VIT. Allergen source is one of critical factors in diagnostic reliability of the BAT, in our study we demonstrated the high specificity of BÜHLMANN CAST Allergens that are also available in Slovakia.

\section{References}

1. Adib-Tezer H, Bayeri C. Primary Paper Wasp Allergy in the Rheingau Area, Germany: More Common than Expected? Aktuelle Derm 2021. DOI: $10.1055 / \mathrm{a}-1310-5058$

2. Poziomkowska-Gẹsicka I, Kostrzewska M, Kurek M. Comorbidities and Cofactors of Anaphylaxis in Patients with Moderate to Severe Anaphylaxis. Analysis of Data from the Anaphylaxis Registry for West Pomerania Province, Poland. Int J Environ Res Public Health 2021; 18, 333. https://doi.org/10.3390/ijerph18010333.

3. Bergmann-Hug K, Fricker M, Hausmann O, Helbling A, Jörg L. Sensitization to Hymenoptera venom in pollen allergic patients: frequency and involvement of cross-reacting carbohydrate determinants (CCD). PLoS ONE 2020; 15(9): e0238740. https://doi.org/10.1371/journal. phone. 0238740 .

4. Krishna MT, Ewan PW, Diwakar L, Durham SR, Frew AJ, Leech AJ et al. Diagnosis and management of hymenoptera venom allergy: British Society for Allergy and Clinical Immunology (BSACI) guidelines. Clin Exp Allergy 2011; 41: 1201-1220.

5. Blank S, Grosch J, Ollert M, Biló MB. Precision Medicine in Hymenoptera Venom Allergy: Diagnostics, Biomarkers, and Therapy of Different Endotypes and Phenotypes. Front Immunol 2020; 11: 579409. DOI: 10.3389/fimmu.2020.579409.

6. Mesquite AM, Carneiro-Leão L, Amaral L, Coimbra A. Hymenoptera Venom Allergy: Re-Sting reactions. Eur Ann Allergy Clin Immunol 2021; 53 (2): 94-96.

7. Adelmeyer J, Pickert J, Pfuetzner W, Moebs C. Long-term impact of hymenoptera venom immunotherapy on clinical course, immune parameters and psychosocial aspects. Allergologie 2021; 44 (2): 139-150. 
778-784

8. Przybilla B, Ruëff F. Hymenoptera venom allergy. JDDG 2010; 8: 114-129.

9. Treudler R, Simon JC. Allergen-specific immunotherapy of hymenoptera allergy: what to consider. Allergologie 2021; 44 (2): 113-119.

10. Trabado AR, Hijón CC, Cantarino AR, Romero-Chala S, GarcíjaTrujillo JA, Pereira LMF. Short-, Intermediate-, and Long-Term Changes in Basophil Reactivity Induced by Venom Immunotherapy. Allergy Asthma Immunol Res 2016; 8 (5): 412-420.

11. Bokanovic D, Arzt-Gradwohl L, Schwarz I, Schrautzer C, Laipold $\mathbf{K}$, Aberer W et al. Possible utility of basophil activation test in dual honeybee and vespid sensitization. J Allergy Clin Immunol Pract 2019; Jun 22. Pii: S2213-2198(19)30559-8. DOI: 10.1016/j.jaip.2019.06.00.8

12. Moitinho Abram D, Romani Fernandes LG, Perez-Riverol A, Brochetto-Braga MR, de Lima Zollner R. Cross-Reactive Carbohydrate Determinant in Apis mellifera, Solenopsis invicta and Polybia paulista Venoms: Identification of Allergic Sensitization and Cross-Reactivity. Toxins 2020; 12, 649. DOI: 10.3390/toxins12100649.

13. Müller UR, Johansen N, Petersen AB, Fromberg-Nielsen J, Haeberli G. Hymenoptera venom allergy: analysis of double positivity to honey bee and Vespula venom by estimation of IgE antibodies to speciesspecific major allergens Api $\mathrm{m} 1$ and Ves v 5. Allergy 2009; 64 (4): 543-548.

14. Stock R, Fischer T, Aßmus K, Zoeller N, Ackermann H, kaufmann $\mathbf{R}$ et al. Safety and tolerability of venom immunotherapy: Evaluation of 581 rush- and ultra-rush induction protocols (safety of rush and ultra-rush venom immunotherapy). World Allergy Organ J 2020; 14:100496, http:// doi.org/10.1016/j.waojou.2020.100496.

15. Hemmings O, Kwok M, McKendry R, Santos AF. Basophil Activation Test: Old and New Applications in Allergy. Curr Allergy Asthma Rep 2018; 18 (12): 77-93.

16. Hoffmann AF, Santos AF, Mayorga C, Nopp A, Eberlein B, Ferrer $\mathbf{M}$ et al. The clinical utility of basophil activation testing in diagnosis and monitoring of allergic disease. Allergy 2015; 70: 1393-1405.

17. Hausmann OV, Gentinetta T, Bridts CH, Ebo DG. The basophil activation test in immediate-type drug allergy. Immunol Allergy Clin North Am 2009; 29 (3): 555-566.

18. Mueller HL. Diagnosis and Treatment of Insect Sensitivity. J Asthma Res 1966; 3: 331-333.
19. Pastorello EA, Borgonovo L, Preziosi D, Schroeder JW, Pravettoni V, Aversano MG et al. Basal Tryptase High Levels Associated with a History of Arterial Hypertension and Hypercholesterolemia Represent Risk Factors for Severe Anaphylaxis in Hymenoptera Venom-Allergic Subjects over 50 Years Old. Int Arch Allergy Immunol 2021; 182 (2): 146-152.

20. Jakob T, Rafei-Shamsabadi D, Spillner E, Müller S. Diagnostics in Hymenoptera venom allergy: current concepts and developments with special focus on molecular allergy diagnostics. Allergo J Int 2017; 26 (3): 93-105.

21. Mortasawi V, Pfuetzner W. Diagnosis of insect venom allergy with special considerations for the significance of skin tests. Allergologie 2021; 44 (2): 120-130.

22. Santos AF, Schreffler WG. Road map for the clinical application of the basophil activation test in food allergy. Clin Exp Allergy 2017; 47 (9): 1115-1124.

23. De Amici M, Barroci F, Caimmi S, Nespoli L, Licari A, Giuliani G et al. Clinical use of basophil activation test in drug, food and hymenoptera venom allergies. Minerva Pediatr 2019; 71 (2): 209-217.

24. Golden DBK. Update on Insect Sting Anaphylaxis. Curr Allergy Asthma Rep 2021; 21 (3): DOI: 10.1007/s11882-021-00998-w.

25. Eberlein B. Basophl Activation as Marker of Clinically Relevant Allergy and Therapy Outcome. Front Immunol 2020; 11:1815. DOI: 10.3389/ fimmu.2020.01815.

26. Aßmus K, Meissner M, Kaufmann R, Valesky EM. Benefits and limitations of Sting challenge in hymenoptera venom allergy. Allergologie 2021; 44 (2): 106-112.

27. Matysiak J, Matuszewska E, Kowalski ML, Kosiński SW, Smorawska-Sabanty E, Matysiak J. Association between Venom Immunotherapy and Changes in Serum Protein-Peptide Patterns. Vaccines 2021; 9, 249. https://doi.org/10.3390/vaccines9030249.

28. Selcuk A, Baysan A. Venom immunotherapy in indolent systemic mastocytosis with high serum tryptase level. Hum Vaccine Immunother 2020; https://doi.org/10.1080/21645515.2020.1846398.

29. Eberlein-König B, Schmidt-Leidescher C, Rakoski J, Behrendt H. In vitro basophil activation using CD63 expression in patients with bee and wasp venom allergy. J Investig Allergol Clin Immunol 2006; 16: 5-10.

Received March 29, 2021. Accepted May 11, 2021. 